\title{
Universal Access in the Information Society
}

\section{A review of assistive spatial orientation and navigation technologies for the visually impaired \\ --Manuscript Draft--}

\begin{tabular}{|c|c|c|}
\hline Manuscript Number: & \multicolumn{2}{|l|}{ UAIS-D-16-00078R3 } \\
\hline Full Title: & \multicolumn{2}{|c|}{$\begin{array}{l}\text { A review of assistive spatial orientation and navigation technologies for the visually } \\
\text { impaired }\end{array}$} \\
\hline Funding Information: & $\begin{array}{l}\text { Fundação para a Ciência e a Tecnologia } \\
\text { (SFRH/BD/89759/2012) }\end{array}$ & Mr. Hugo Fernandes \\
\hline Corresponding Author: & \multicolumn{2}{|c|}{$\begin{array}{l}\text { Hugo Fernandes, Ph.D } \\
\text { INESC TEC and University of Trás-os-Montes e Alto Douro } \\
\text { PORTUGAL }\end{array}$} \\
\hline First Author: & \multicolumn{2}{|l|}{ Hugo Fernandes, Ph.D } \\
\hline \multicolumn{3}{|l|}{ First Author Secondary Information: } \\
\hline \multirow[t]{5}{*}{ Order of Authors: } & \multicolumn{2}{|l|}{ Hugo Fernandes, Ph.D } \\
\hline & \multicolumn{2}{|l|}{ Paulo Costa, MSc. } \\
\hline & \multicolumn{2}{|l|}{ Vitor Filipe, Ph.D } \\
\hline & \multicolumn{2}{|l|}{ Hugo Paredes, Ph.D } \\
\hline & \multicolumn{2}{|l|}{ João Barroso, Ph.D } \\
\hline \multicolumn{3}{|c|}{ Order of Authors Secondary Information: } \\
\hline Author Comments: & \multicolumn{2}{|c|}{$\begin{array}{l}\text { Thank you very much for the revisions you sent on our paper. } \\
\text { We have adopted the recommendations made by the reviewers, resulting in changes } \\
\text { throughout the document, strongly improving our work. }\end{array}$} \\
\hline Response to Reviewers: & \multicolumn{2}{|c|}{$\begin{array}{l}\text { The authors would like to thank the reviewers and the editor for all the cooperation and } \\
\text { assistance. Your contributions significantly improved the manuscript and our work }\end{array}$} \\
\hline
\end{tabular}


methodology. Our sincere thanks.

Powered by Editorial Manager ${ }^{\circledR}$ and ProduXion Manager ${ }^{\circledR}$ from Aries Systems Corporation 


\begin{abstract}
Purpose - The overall objective of this work is to review the assistive technologies that have been proposed by researchers in recent years to address the limitations in user mobility posed by visual impairment.

Method - This work presents an "umbrella review".

Results - Visually impaired people often want more than just information about their location and often need to relate their current location to the features existing in the surrounding environment. Extensive research has been dedicated into building assistive systems. Assistive systems for human navigation, in general, aim to allow their users to safely and efficiently navigate in unfamiliar environments by dynamically planning the path based on the user's location, respecting the constraints posed by their special needs.

Conclusions - Modern mobile assistive technologies are becoming more discrete and include a wide range of mobile computerized devices, including ubiquitous technologies such as mobile phones. Technology can be used to determine the user's location, his relation to the surroundings (context), generate navigation instructions and deliver all this information to the blind user.
\end{abstract}




\section{INTRODUCTION}

The World Health Organization (WHO) [1] reported in 2013 that 285 million people are estimated to be visually impaired worldwide: 39 million are blind and 246 million suffer from low vision. From the overall population with visual impairment, about $90 \%$ of the world's visually impaired live in developing countries and $82 \%$ of people living with blindness are aged 50 and above. Regrettably, this percentage is expected to increase in the coming decades. Visual impairment has a significant impact on individuals' quality of life, including their ability to work and to develop personal relationships. Almost half (48\%) of the visually impaired feel "moderately" or "completely" cut off from people and things around them [2].

There are four levels of visual function, according to the International Classification of Diseases (ICD 10, Update and Revision 2006): normal vision, moderate visual impairment, severe visual impairment and blindness [3]. Moderate visual impairment combined with severe visual impairment may be grouped under the term "low vision"; low vision combined with blindness represents all forms of visual impairment [1]. In order to overcome or lessen the difficulties imposed by visual impairment, extensive research has been dedicated to building assistive systems. The need for assistive technologies has long been a constant in the daily lives of people with visual impairment and will remain so in future years. There are various definitions for assistive technology in general. Common to all of them, however, is the concept of an item or piece of equipment that enables individuals with disabilities to enjoy full inclusion and integration into society [4-6]. Traditional assistive technologies for the blind include white canes, guide dogs, screen readers, and so forth. However, the detectable ranges of white canes are very short (at most 1.5 meters) and, consequently, the visually impaired can only immediately detect nearby obstacles at ground level. Guide dogs are also used by the visually impaired to navigate to their destinations avoiding the dangers they may encounter along their path. However, it is difficult to provide a sufficient number of guide dogs because of the long-time periods needed to train them, as well as the high costs associated with their training. Furthermore, it is also quite difficult for the visually impaired to take care of the living dogs appropriately [7].

Modern mobile assistive technologies are becoming more discrete and include (or are delivered via) a wide range of mobile computerized devices, including ubiquitous technologies like mobile phones. Such discrete technologies can help alleviate the cultural stigma associated with the more traditional (and noticeable) assistive devices [8].

Visual impairment imposes many restrictions and specific requirements on human mobility. The overall objective of this work is to review the assistive technologies that have been proposed by researchers in recent years to address the limitations in user mobility resulting from visual impairment. This work does not focus on the analysis and description of individual systems. Instead it will review how technology is being used in recent years to individually address the different tasks related to assistive human navigation and how the components of traditional navigation systems can be adapted to address the limitations and requirements of visually impaired users. Human navigation, in general, requires an estimation of the user location, the relation to its 
context and finding a way to a specific destination. This work will cover these topics in dedicated sections. In this work, the term "visual impairment" incorporates any condition that impedes an individual's ability to execute typical daily activities due to visual loss. Because the aim of this work is to present a general review of navigation and orientation assistive technologies for the visually impaired, low vision is not separated from total blindness and so these terms are used interchangeably.

\section{UNDERSTANDING HUMAN NAVIGATION}

Human beings have the ability to acquire and use information obtained from the surrounding environment using their natural sensors. They have developed a number of evolutionary mechanisms that enable the distinction between different objects and the triggering of events and complex processes based on their perception of reality.

Cognition concerns knowledge and knowing in intelligent entities, especially by human beings, but also nonhuman animals and synthetic computational entities such as robots [9]. Cognition includes the mental structures and processes involved in perception, attention, thinking and reasoning, learning, memory, linguistic and nonlinguistic communication. It also includes external symbolic structures and processes, such as maps or written procedures for carrying out formal spatial analysis, which assist internal cognition. Similarly, cognition is often about space, place, or environment, so cognitive acts are quite often of geographic nature [9].

Cognitive mapping [10] is of extreme importance for individuals in terms of creating a conceptual model of the surrounding space and objects around them, thereby supporting their interaction with the physical environment [11]. In new environments, finding your way can be time consuming and may require a considerable amount of attention. In these types of scenario visual impairment is a major limitation to user mobility. On one hand, individuals with visual impairments often need the help of sighted people to navigate and cognitively map new environments, which is time consuming, not always available and leads to lower mobility [12]. On the other hand, individuals with cognitive impairment may experience difficulty in learning new environments and following directions.
Assistive systems for human navigation generally aim to allow their users to safely and efficiently navigate in unfamiliar environments, without getting lost, by dynamically planning the path based on the user's location, respecting the constraints posed by their special needs. Collecting the specific needs or specificities of any impairment is a key point for the development of any assistive system. Using direct observational and interviewbased knowledge elicitation methods, researchers of The Haptic Sight study [13] tried to gain a better understanding of a visually impaired person's indoor walking behaviour and the information required for him to walk independently. They found that the visually impaired need to be aware of their current location, the direction they are heading, the direction they need to go and the path to their destination. Only after the research team had identified these parameters did they develop a handheld device-based application. In other words, users with visual impairment must be aware of their physical location, their relation to the surrounding environment (context) and the route they must follow to navigate to a desired destination. When designing an assistive system for human navigation, separate processing units (or modules) can address these identified tasks, namely: Location, Orientation, Navigation and Interface, as seen in Figure 1.

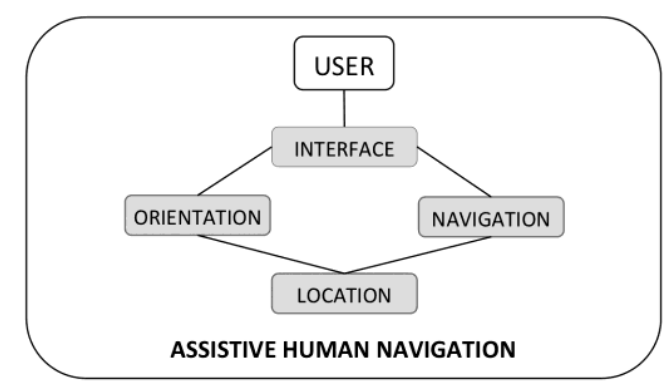

Fig. 1: Units addressing the tasks required for assistive human navigation.

This work reviews different ways with which different researchers addressed the use of technology to fill the gaps and needs presented by visual impairment in each of these topics. As with the design of any assistive system, the interface with the user must be adequate to the user's limitations. This work will cover this topic in a dedicated section as well. 


\section{LOCATION}

All guidance/navigation systems must include a basic form of localization, i.e., the determination of a user's location and/or pose. The estimation of the user's location is sometimes referred to as 'positioning' [14]. The most common localization methods can be grouped into four different categories: (1) direct sensing, (2) dead reckoning, (3) triangulation, and (4) pattern-recognition [15]. It is important to understand that depending on the technology used, the user location may be estimated by the direct application of techniques, or by using computational methods to process data that can indirectly contribute to estimate the location. It is also important to distinguish between the two. If, on one hand, direct sensing techniques can almost directly provide an indication of the user's location, other methods, such as dead reckoning, use the components of locomotion (heading, acceleration, speed, etc.) to computationally estimate the displacement from a known location. The same applies to triangulation and pattern-recognition. In the case of pattern recognition, it is not the actual detection of the visual pattern that provides an estimation of the location. Instead, some of the metrics and data outputting from the detection (such as pose and distance from the detected pattern) can be used to computationally make the estimation. The location can be used for both planning the path (navigation) and providing surrounding (contextual) information (orientation). If the user's location is known, the system can also find a new path in case the user gets lost or calculate an alternative path, if needed. The planned path is then used to generate and provide guiding directions to a user-specified destination.

\subsection{Direct sensing techniques}

Localization techniques based on direct sensing determine the location of the user through the sensing of identifiers (or tags), which have been installed in the environment.

Typical direct sensing technologies include the use of Radio Frequency Identification (RFID) tags that can either be passive [16-19] or active [20] (some systems use both active and passive tags [21,22]), infrared (IR) transmitters that are installed in known positions where each transmitter broadcasts a unique ID [23,24], Bluetooth beacons [25] or visual barcodes [26,27]. All of these technologies require the user to carry extra equipment to sense the identifiers. In the case of radio-frequency identification, though single RFID tags are quite inexpensive, in order to massively install them in large environments may become costly. Another disadvantage is the range of detection. In the case of passive tags the range is too low. In the case of active tags the range is higher but they require individual power supply (and respective maintenance). Infrared emitters require the user to be in the line-of-sight and, even so, they are strongly affected by sunlight interference. Bluetooth beacons, when used for localization, require the user to walk more slowly than with other sensing techniques because of the communication/pairing delay. Barcodes are, in a way, very similar to radiofrequency identification. This approach is low cost, easy to install and to maintain. The main limitation is that the user has to find each barcode and scan it, which may be cumbersome and will slow down navigation. In the case of blind users, using a system that searches for printed barcodes that they cannot see is also very demanding and prone to reading failure.

\subsection{Dead-Reckoning}

Humans maintain (update) their sense of orientation as they move around via a combination of two processes, i.e. landmark-based and dead reckoning processes. Landmark-based updating involves recognizing specific features in the world that may be associated with known places. Dead reckoning updating involves keeping track of the components of locomotion (including heading, velocity or acceleration) and travel duration. Dead reckoning is sometimes referred to as "path integration' [15].

While the user is moving, a dead-reckoning system estimates the user's location through a combination of odometry readings. Odometry readings can be acquired through a combination of sensors such as accelerometers, magnetometers, compasses, and gyroscopes [28-31] or using a user's specific walking pattern (such as the user's average walking speed) [32].

An initial location is typically determined using a global navigation satellite system (GNSS) like the Global Positioning System (GPS)[29], Radiofrequency identification (RFID) tags [30], or cellular phone positioning (GSM broadcasting stations) [31]. Since the location estimation is a recursive process, inaccuracy in location estimation translates into errors that accumulate over time. The accumulated error can be corrected using environmental 
knowledge. The users' position can be synchronized using periodic updates from direct sensing localization techniques such as RFID tags, or pattern-matching localization methods such as the use of data extracted from the recognition of known visual landmarks. A benefit of processing data from pattern-matching over direct sensing techniques is a lower installation cost, as a smaller number of identifiers must be installed.

\subsection{Triangulation}

Though most direct sensing techniques try to locate the user by sensing one unique identifier, several systems employ multiple identifiers and use triangulation computational methods to locate the user. These methods locate the user by triangulating the sensed tags installed in known locations. The tags that have been frequently used for indoor or outdoor localization include RFID [33], Infrared (IR) [34,23], and ultrasound [35,36].

Lateration uses the distance between the user and at least three known points, whereas angulation uses the angular measurements from at least three known points to the user to determine the users' location [37]. Global Positioning System (GPS) is the most commonly used system for outdoor localization [38-40] and uses a trilateration computational method to locate the user, based on known satellite positions. GPS receivers analyze a periodic signal sent out by each satellite to compute the latitude, longitude, and altitude at the users' position. For outdoor navigation, GPS has become the standard as it is free, reliable, and it is available any place on Earth in any weather condition. The main disadvantage of GPS localization is that the GPS signal strongly degrades inside buildings, between tall buildings or in dense forest areas (such as parks). There are two alternative triangulationbased techniques, which are available in contexts where GPS signals are not sensed, or available. Cell-tower positioning [41] uses the triangulation of the known locations of cell towers with the provided signal strength of each cell phone tower, whereas wireless local area networks (WLAN) positioning [42,24] triangulates the location of wireless base stations using the signal of each emitting station. Both techniques have a lower precision than GPS due to multi path reflection problems.

Another way of using the signal from wireless emitting stations, such as $\mathrm{Wi}-\mathrm{Fi}$, is signal fingerprinting. This approach is based on signal strength observations on previously known locations. An estimate of the location is obtained based on these measurements and a signal propagation model. The propagation model can be obtained by simulation or with prior calibration measurements in certain locations. In this last case, the measured signal strength values at a certain location are compared with the signal strengths values of pre-calibrated points stored in a database. This approach, with proper calibration, can provide extremely high accuracy, in comparison with GNSSbased approaches and has been successfully adopted in the field of robotics and unmanned vehicle applications. The major limitation in its application on the blind user case is the cost-overbenefit. The required time and costly signal strength system calibration is very high in the beginning $[43,44]$.

\subsection{Pattern Recognition}

Recently, systems have been developed which use Computer Vision techniques, like pattern matching, to sense the surrounding environment and detect visual landmarks. Although at first glance it may be quite obvious that pattern-recognition alone cannot provide an indication of the user location, an estimation can indirectly be extracted using the outputting data from the pattern detection, such as pose and distance to the detected pattern. The most common artificial vision systems developed to support the guidance of blind users extract this type of information by analyzing the characteristics of the objects detected in the captured image using classical image-processing techniques $[45,46]$. Some systems go further by combining vision sensors with positioning sensors or even combining multiple vision sensors to obtain a $3 \mathrm{D}$ representation of the scene (to obtain depth information).

\subsubsection{Motion and pose estimation}

Systems that use computer vision to estimate the location and orientation of the user, enable him/her to perceive their relative position to a detected georeferenced visual landmark [47-49]. When the user is carrying a camera whose position and orientation relative to the user's body are known, the motion of the features detected in the captured images may be used to assess information about the carrier's pose and motion. Visual motion 
information is not affected by the same error sources as global navigation satellite systems or selfcontained sensors (like inertial sensors), and is therefore a complementary information source for increasing the accuracy of the positioning measurements [50]. Research related to visual positioning methods has been mainly focused on the autonomous navigation of vehicles and mobile robots.

The first papers related to the use of computer vision assistance in pedestrian navigation were published in the late 90s [51]. They described the use of databases preloaded with images of samples taken of the expected surroundings, which were tagged with information about their geographic location. The position of the pedestrian was provided when a match was found between an image taken by the pedestrian and an image stored in the database [52]. The database and the image processing could be made locally or remotely on a server, depending on processing power requirements [53].

A visual pedestrian navigation system independent of a server and of pre-existing databases usually needs integration with other positioning sensors to be functional. In such a system, monitoring the motion of features in consecutive images taken by the user device and integrating the information with measurements obtained with other sensors or a Global Navigation Satellite System (GNSS) receiver can be used to obtain the relative position of the user. Initial absolute position information can be used to reduce drift and other errors, as without initial position the visual perception only provides information about the user's motion. Such serverindependent systems have been proposed [54] using visual-aided Inertial Measurement Unit (IMU) measurements. Other techniques, like the ones used in Simultaneous Localization and Mapping (SLAM) systems, produce a map of the unknown environment while simultaneously locating the user. Traditionally, mapping has been done using inertial sensors, though in recent years SLAM systems that also integrate a camera (visual SLAM systems) have been developed [55].

The magnitude of the motion of a figure in an image is dependent on the relative depth of the object within the captured scene, i.e. the distance of the object from the camera. Because the distance of objects from the camera in the environment is usually unknown, a scale problem arises and different methods for overcoming it have been used
[50]. Tools for resolving the distance, like laser rangefinders, have been integrated with a camera [56]. The requirement for carrying special equipment reduces the applicability of this method for pedestrian navigation, especially for blind users. Another approach is the use of computer vision algorithms to detect artificial landmarks with known indoor location (georeferenced landmarks). Recently, indoor navigation systems have been proposed, which use computer vision to detect and decode fiduciary markers in real-time, using standard camera phones. One of the most common markers used are 2-D barcodes. The barcode provides a unique ID and a fixed sized template, which may be used to estimate the pose of the viewer [57]. Using these types of special markers, a standard smartphone may be used in these kinds of systems, without the need to carry any extra equipment. Once the marker is in the camera's field of vision, the user can receive a warning about his relative bearing to the marker, as well as an approximate distance.

\subsubsection{D Sensing}

Distance is one of the most important aspects of navigation, as it is used to avoid collisions or recognize nearby objects. The way human vision uses different perspectives of the same scene to create a three-dimensional perception of the world inspired the use of multiple cameras to model/recognize the world in three dimensions. When a stereo camera is used, the distance to objects may be estimated using triangulation [58]. In the case of stereovision, the distance between the two cameras, called the baseline, affects the accuracy of the motion obtained from the images. The farther the two cameras are from each other, the better the accuracy will be [59]. Stereovision may be used to obtain 3D range information and area correlation methods can be used for approximate depth information. This information has been successfully used in combination with pedestrian detection models [60]. Methods using genetic algorithms have been used to perform stereovision correlation and generate dense disparity maps, as well. These disparity maps, in turn, provide rough distance estimates to the user, allowing them to navigate through the environment [61]. Simpler approaches use one relative view (right or left camera) and a depth map (from the stereo vision equipment) to perform fuzzy-based clustering segmentation of the scenario into object 
clusters [62]. After, knowing the clusters' locations, it is possible to detect near and far obstacles and feed this information to the user. The detection of changes in a 3D space based on fusing range data and image data captured by the cameras may also be used to create a $3 \mathrm{D}$ representation of the surrounding space that can be transmitted to the user through an appropriate interface, namely haptic [63].

Having a short-term depth map computed about the user's immediate environment may be used to classify the path/scene as having (or not) any immediate obstacles, whether they are ground based, aerial or their relative position (left/right).

Recently, 3D vision sensors have evolved considerably and have been applied in several popular devices such as smartphones and game consoles, greatly reducing its cost.

Stereovision has been successfully applied to mobile devices (smartphones) allowing the structure of the environment to be estimated and for some kind of obstacle classification to be performed [64]. Theoretically, stereovision camera phones can work and be used to extract the same type of information as other standard stereovision systems. In the case of the use of smartphones, the main limitation is their low processing power in terms of real time execution. In recent years, it has been significantly increased. However real world scenes are usually very structured and obstacle classification in real time is still only used to work as a virtual stick, or white cane (not replacing it entirely, for security reasons).

In many cases, stereoscopic vision has been replaced by the Microsoft Kinect sensor [65]. This led to the mass use of these sensors in scientific research with good results [66-68]. The Kinect sensor includes a depth sensor and an RGB camera. The depth sensor is composed by an infrared laser source that projects non-visible light with a coded pattern combined with a monochromatic CMOS image sensor that captures the reflected light. The pattern received by the RGB sensor is a deformed version of the original pattern, projected by the laser source and deformed by the objects on the scene. The algorithm that deciphers the light coding generates a depth image representing the scene. Using machine-learning techniques, such as neural networks, to analyse depth images obtained from the Microsoft Kinect sensor enables the recognition of pre-defined features/patterns of the surrounding environment [69]. Generally, in terms of the contribution that data extracted from computer vision pattern recognition can give to location systems, whether using stereovision or other image-based sensors like the Kinect, distance can be estimated and, in combination with data from pattern/feature detection and an appropriate geographic information system, contribute to assess the location of the user.

In this context, data for vision-based localization must also be present in the geographic information system used. The geographic information system is a central element to provide any type of locationbased service, and its importance is discussed further in this paper.

\subsection{Summary}

All the technology referred to in the previous sections can be summarized as seen in Table 1.

Table 1: Summary of technology application scenario

\begin{tabular}{|c|c|c|c|c|}
\hline & & Indoor & Outdoor & $\begin{array}{c}\text { Needs } \\
\text { Infrastructure }\end{array}$ \\
\hline \multirow{4}{*}{ Direct-sensing } & RFID & $\bar{\checkmark}$ & $\bar{\checkmark}$ & $\checkmark$ \\
\hline & Bluetooth & $\checkmark$ & $\checkmark$ & $\checkmark$ \\
\hline & $\mathrm{IR}$ & $\checkmark$ & - & $\checkmark$ \\
\hline & Barcodes & $\checkmark$ & $\checkmark$ & $\checkmark$ \\
\hline $\begin{array}{c}\text { Dead- } \\
\text { reckoning }\end{array}$ & IMU & $\checkmark$ & $\checkmark$ & - \\
\hline \multirow{3}{*}{ Triangulation } & $\begin{array}{l}\text { from direct- } \\
\text { sensing }\end{array}$ & $\checkmark$ & $\checkmark$ & $\checkmark$ \\
\hline & GNSS & - & $\checkmark$ & - \\
\hline & Fingerprint & $\checkmark$ & $\checkmark$ & $\checkmark$ \\
\hline \multirow{3}{*}{$\begin{array}{l}\text { Pattern- } \\
\text { recognition }\end{array}$} & Markers & $\checkmark$ & $\checkmark$ & $\bar{\checkmark}$ \\
\hline & $\begin{array}{c}\text { Natural } \\
\text { elements }\end{array}$ & $\checkmark$ & $\checkmark$ & - \\
\hline & 3D sensing & $\checkmark$ & $\checkmark$ & - \\
\hline
\end{tabular}

Using different sensors as an input for estimating the geographical location of the user generates a high degree of redundancy, assuring that the location is always available, reducing the dependence on the infrastructure (as it is with the case of separate use of RFID, fiducial markers or GPS). This type of location system would provide a base layer on which all the remaining locationbased services (such as orientation and navigation) would rely on (Fig. 1). 


\section{SPATIAL ORIENTATION}

Visually impaired people often want more than just information about their location, having the need to relate their current location to the features existing in the surrounding environment.

Orientation and mobility are essential skills for performing a proper navigation [70]. In this process, mobility, or micro navigation, relates to obstacle detection and avoidance in the immediate physical environment. Orientation, or macro navigation, translates as the ability to create and maintain awareness of one's position in the physical space relative to both the landmarks in the surrounding environment, whether they are points of interest (POI) or obstacles (from micro navigation), and to the user's desired destination [71].

A wide range of systems and tools is available for enhancing the mobility of visually impaired individuals. The white cane and the guide dog are the most popular. The white cane is the simplest, cheapest, most reliable and the most popular. However, it does not provide all the necessary information of context such as speed, volume and distances. The eyes usually gather this information, which is necessary for the perception and control of locomotion [72]. Several approaches have been conducted over the last decades to address problems relevant to blind mobility and context awareness. They can be classified into two main categories. 'Electronic Travel Aids' (ETAs) are designed to improve mobility by detecting obstacles in the user's surroundings. In order to improve the blind user's autonomy, 'Electronic Orientation Aids' (EOAs) provide the blind with some degree of situational awareness and guidance in unknown environments [73].

Apart from a few implementations that use some of the location techniques described in the previous section, up to now, EOAs have mainly been based on GNSS and Location-Based Services. However, in recent years, computer vision techniques have successfully been used to provide contextual awareness and orientation indications. In general, these assistive orientation systems use computer vision techniques to provide information ranging from the simple presence of obstacles, or the distinction between fixed and moving obstacles, to the recognition of specific objects in the captured image. In some cases, even the distance and relative displacement of the detected objects to the user is provided, using depth information.
Although very simple in their purpose, systems designed to provide the blind user with information about the existence of objects in his path (through the use of artificial vision sensors) use a wide range of techniques to analyze the image. Traditional image processing techniques can be used to detect the contours of objects in the scene [74,75,47]. More advanced approaches use artificial intelligence techniques to detect obstacles in the captured image [76] and even to classify the scene, presenting basic forms of characterization/description of the environment as being very cluttered or relatively broad [77]. Other classification methods may provide information regarding the spatial distribution of the obstacles/objects in the scene [49], achieving the overall objective of providing direct specific orientation instructions and simple contextual awareness.

More advanced systems, which apply object recognition algorithms to detect and recognize specific objects in the scene, go even further trying to reduce the gap between sighted and non-sighted people. Using their natural sensors, sighted users not only detect the existence of objects and obstacles in their immediate surroundings, but they are also able to recognize them and their attributes, such as color, shape and relative spatial orientation. The simplest approaches use markers placed at specific points-of-interest [78]. When detected, these markers are used to estimate the user location and, subsequently, the objects that are expected to be found on the scene. Additionally, it is also possible to inform the user about the distance and relative position to the marker (pose). However, the most common systems that use object recognition to provide contextual information try to locate and recognize natural objects in the scene without the need to use artificial markers placed in the infrastructure. As discussed in earlier subsections, the placement of markers/sensors in the infrastructure is costly and requires a lot of maintenance. Given this fact, many assistive systems nowadays try to give the user information about the presence and orientation of natural objects in the scene, such as crosswalks [79-81] or text commonly found in places like Buses or office doors [82,83,48]. Even the distinction between similar objects used in everyday life that may be easily confused by blind users, like different bank notes [84], food or medicine containers [85], can be incorporated in spatial orientation systems which use advanced computer vision techniques to provide 
Table 2: Summary of the features provided by the most common spatial orientation assistive devices

\begin{tabular}{|c|c|c|c|c|}
\hline & \multicolumn{2}{|c|}{ Traditional } & \multicolumn{2}{|c|}{ Electronic } \\
\hline & $\begin{array}{l}\text { White } \\
\text { cane }\end{array}$ & $\begin{array}{c}\text { Guide } \\
\text { dog }\end{array}$ & $\mathrm{EOA}$ & $\begin{array}{l}\text { Comp. } \\
\text { Vision }\end{array}$ \\
\hline Environment & $\begin{array}{l}\text { Indoor/ } \\
\text { Outdoor }\end{array}$ & $\begin{array}{l}\text { Indoor/ } \\
\text { Outdoor }\end{array}$ & Outdoor & $\begin{array}{l}\text { Indoor/ } \\
\text { Outdoor }\end{array}$ \\
\hline Speed & - & - & $\checkmark$ & - \\
\hline Distance & $\checkmark$ & - & $\checkmark$ & $\checkmark$ \\
\hline Obstacles & $\checkmark$ & $\checkmark$ & $\checkmark$ & $\checkmark$ \\
\hline $\begin{array}{l}\text { Object } \\
\text { recognition }\end{array}$ & - & - & - & $\checkmark$ \\
\hline $\begin{array}{l}\text { Environment } \\
\text { description }\end{array}$ & - & - & $\checkmark$ & $\checkmark$ \\
\hline
\end{tabular}

With the recent advances in $3 \mathrm{D}$ vision and depth sensors, an all-new kind of contextual input may be used in the context of assistive systems for the visually impaired: depth information. Using feature descriptors and machine learning techniques, different objects can be extracted and classified [86]. These types of systems can recognize threedimensional objects from the depth data, and inform visually impaired users not only about the existence of objects but also their class, such as chairs and upward stairs [87,7,88], working similarly to a conventional white cane, with an extended range. Some systems even incorporate the detection and distinction between fixed and moving obstacles and object recognition in one global solution, mostly for pedestrian detection and avoidance [89-92].

\section{NAVIGATION}

The term 'navigation' defines the behaviour of moving towards a destination, with all the motor,

sensory, and cognitive processes that it implies [70]. Downs and Stea define navigation as "the process of solving one class of spatial problems, the movement of a person from one location on the earth's surface to another" [93]. They divided the process into four tasks: orienting oneself in the environment, choosing the route, keeping on track and recognizing that the destination has been reached. Human navigation is performed using a combination of mobility and orientation [94]. In general, human navigation in indoor and outdoor environments is performed by measuring the distance and relative orientation to one, or multiple, reference points (context). People employ either path integration, orienting themselves relative to a starting position, or landmark-based navigation, where they rely upon perceptual cues together with an external or cognitive map. Humans may also use a combination of both path integration and landmark-based navigation [95]. A number of features in the environment can be used to help determine the location. To maintain a sense of where they are in such situations, humans rely on their estimates of the direction and velocity of movement obtained from their vestibular, proprioceptive, and kinesthetic senses, here referred to as path integration [96].

In the case of path integration, a single reference point is used throughout the navigation, and the location is estimated based on the addition of all the changes in position and orientation [97].

In the case of landmark-based navigation, users change from reference point (landmarks) to reference point as they navigate in the environment, considering the relative position of the landmarks. In this case, a physical or cognitive map of the environment is used. By periodically measuring the displacement and changes in the orientation (based on heading and motion) and combining them with the distance and orientation relative to a reference point, such as a landmark, users can estimate their new location and orientation while navigating in an environment.

A powerful assistive device combines both micro navigation (sensing the immediate environment) and macro navigation (reaching a remote destination) functionalities. The micro navigation functions serve to restore a set of sensorimotor behaviors based on visual object localization (context). The macro navigation functions provide 
the user with global orientation and navigation skills [70].

All navigation systems have three functional components: an input module to determine the location and orientation in space, a spatial database of the environment and an interface, which delivers information to the user. Location information is usually obtained using the individual location technologies discussed in a previous section, or by a fusion or combination of different inputs, including computer vision. These three components are used as well in the case of navigation systems designed for the visually impaired [98].

The location can be used for both planning the path and providing contextual information. The advantage of using a navigation system to plan a route is that the path can be optimized based on different concurring paths and specific user requirements, such as shortest or safest path. In the case of individuals with vision impairments, a path that goes along walls reduces the chance of the user getting lost and a path that avoids low ceilings is much safer [15].

In this context, a geographic information system (GIS) designed to enable all these assistive features must provide ways to store and deliver data of much broader extent than simple points-of-interest and POI categorization. An appropriate geographic system is a core element in any navigation system. Path planning algorithms use graphs or grids to represent the environment. These elements must also be stored in the GIS. To plan a path using graph-based approaches, the environment is divided into sets of nodes and edges connecting these nodes. Edges connect nodes based on the environment map and if one node is accessible from the other one. In this case, each edge may have a weight assigned to it based on different criteria for the path planning. A graph-based approach has the advantage of creating the nodes only if there are objects. Edges are created only if objects are accessible from each other. In complicated environments with many objects, the graph may become big and decrease the performance of the path-planning algorithm. The weight associated with edges or cells plays an important role when customizing a path. For example, in the case of a path that should avoid stairs, the edges with stairs receive higher weights, and edges with low ceiling have higher weights when planning a path for individuals with visual impairments. Most of the current navigation systems use either Dijkstra $[29,30,99,100]$ or $A^{*}[29,101,32]$ for path planning.

\section{INTERFACE}

Accurate recognition and distinction between the contextual elements found in the environment, whether by using computer vision or any other form of input is of the highest importance on an EOA device. Interfacing with the user to provide information about the elements found in the scene is also crucial, as the interpretation of the reality around the user directly influences his safety and, ultimately, the adoption of this kind of assistive technology. The most commonly found ways of interfacing with the user nowadays are sonification, audio description and haptic interfaces. These are the most commonly found ways to interface with an electronic assistive system for the blind, and its use is valid to both receiving alerts about the physical elements detected, as well as to receive and understand wayfinding instructions.

Sonification is, by definition, the use of non-speech audio to convey information or perceptualize data. The use of acoustic (sound/sonification) patterns to provide this information to the user is very common among EOAs for the visually impaired $[76,74,46]$. In some cases, sonification is even used to provide the relative position of the detected obstacles $[49,102]$. These systems use 3D audio (Fig. 2) to provide audio cues that can be perceived as if they were being generated by the detected landmark. The concept behind 3D audio is the use of different sound sources, located at different locations to provide the feeling of directional hearing (Fig. 2).

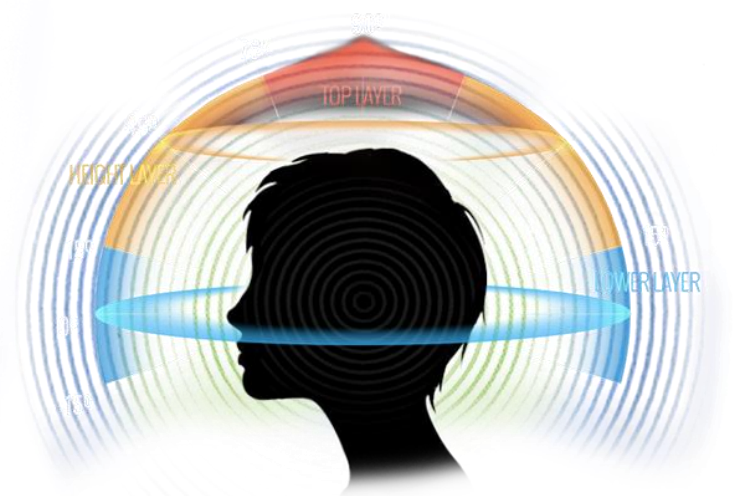

Fig. 2: Perceptual feeling provided by 3D audio (http://www.auro-3d.com/consumer/).

The most obvious advantage of adding spatial sound modelling to audio interfaces over sequential 
techniques is the natural perception. Individuals without hearing impairment use their directional hearing for orientation at all times [103]. This kind of interface can be used to provide simple, yet immediately perceivable cues about bearing or relative position (pose) to an obstacle. The fact that blind people often rely on audio cues from the environment for orientation creates restraints on using headphones for acoustic feedback. Alternatives like bonephones are viable [104].

Audio description has the same considerations as the sonification methods. According to [105] "one major issue to be considered in the design of an interface is whether a rich description of the scene, or only highly symbolic information, should be provided to the user".

Another approach is to present the information about the obstacles detected in the image through the use of haptic interfaces [106,75,7]. 3D range data may be converted into a $2 \mathrm{D}$ vibrating array attached to the user's body [63]. With appropriate signal coding, the use of $2 \mathrm{D}$ vibrating patterns can reproduce depth information. Haptic interfaces are also used in a way in which an array of pins works in a similar way as Braille display [107].

Some other, less usual, forms of interface are still in investigational devices, not available for commercial use. One example [108] consists of a camera (mounted in sunglasses), one transducer and a postage-stamp-size electrode array that is positioned on the top surface of the tongue. The recorded images are translated into gentle electrical signals and displayed on the tongue. The generated stimulation patterns reflect key features of the recorded images like high contrast objects and their movements.

As a general consideration, any of the user's remaining sensory channels (tactile or acoustic) can be used. However their use should be carefully considered as it may interfere with performing other tasks that the blind users cannot do without. The amount of information to be presented to the user should be carefully considered as well, as information capacity of the nonvisual senses is much smaller than vision [109]. The cues provided by these interface channels represent the most common ways of interfacing with assistive devices, for the blind, and provide the means to understand the information generated that can be used whether for context description or wayfinding.

\section{CONCLUSIONS}

This work presented a general review of navigation and orientation assistive technologies for the visually impaired. As with the development of any other assistive technology, the development of these types of systems must be centred on the specific needs and restrictions of the user and his impairment. The use of an assistive system must not pose any interference with everyday life activities or it will be considered as an obstacle itself. If this happens, the community will not adopt the system. Sometimes the technologies used are not perfectly adequate or do not meet specific needs, either due to its mode of operation, or to the way they convey information to the user.

In terms of location systems, despite the efforts to create cheap, reliable and accurate positioning systems, it has been difficult to find a system with a good balance between these three factors. Of the various technologies used to locate the user, the most common are RFID, GPS, Wi-Fi and Computer Vision. The use of RFID proves to be very accurate. However, from the user's point of view alone, it is unreliable because the user easily gets lost in case of failure in reading multiple successive tags. This can happen due to the long reading times required (in relation to the white cane swipe movement) and/or low tag density. From the point of view of implementation, despite its low cost (compared to the benefit), such a system requires extensive maintenance. Although, ideally most places could benefit from installing such systems, in practice the only places that will consider their use are those where the relationship between benefit over cost is attractive. These places can include shopping areas, public libraries, etc. The use of GPS has enough accuracy for most users without any special need; it is free and covers the earth's entire surface. However, its availability inside buildings is very low and, even in places with good reception of GPS signal, common systems based on smartphone, or cheap commercial antennas, do not offer the precision needed to safely guide a blind user. Often, an error of 5 meters is the difference between being in the sidewalk, or the in the middle of the road. The use of Wi-Fi is very similar to RFID in the aspect that the characteristics of a source (georeferenced) signal are used to assess or estimate the user's location. As in the case of RFID, it may work on exterior and interior environments. It has the great 
advantage of having more range than RFID, though suffers from serious accuracy problems due to problems with signal reflection (multiple path). In the case of the use of Computer Vision, this technology can cover all types of environments, indoors and outdoors and its accuracy is sufficient; however, it has some limitations. If the system is based on recognizing known artificial landmarks (fiducial markers), an infrastructure management effort is required, as well as a large information system to support the georeferenced data. In the cases when natural landmarks are used, object recognition involves the use of more complex algorithms, more prone to errors, which may limit real-time operation. The most successful systems resort to the combined use of various technologies, either in a redundant manner or in a complementary way. Combining the occasional use of these direct-sensing technologies for error correction (or minimization) and the permanent use of inertial measurements for constant location estimation, such a multisensorial location system would allow for the creation of a reliable and "always available" location system, increasing the trust of the users concerning these types of assistive technologies, while reducing installation costs and infrastructure dependency. From the summary of the variety of techniques, methods and technologies that may be used to assist a blind user on knowing his location, a powerful location module would be one that could combine all the different types of possible location inputs and fuse them into a unique, hybrid, location system based on a multisensorial approach. Using different sensors as an input for estimating the geographical location of the user generates a high degree of redundancy, assuring that the location is always available, reducing the dependence on the infrastructure (as it is with the case of separate use of RFID, fiducial markers or GPS). This type of location system would provide a base layer on which all the remaining locationbased services (like orientation and navigation) would rely on.

With regard to orientation systems, for user guidance/contextualization, most of them try to somehow extract context information and provide more information to the user than just his location. The location input can be used to provide contextual information (orientation or micro navigation), either about the features of the surrounding environment, or about the services available in the premises. A key factor in that respect is the structuration of the information stored in the GIS. To be useful, the information must be transmitted to the user in a manner in which it is easily understood, using a natural language as interface. To achieve this goal, the user must be able to define (and configure, in real time) a certain degree of awareness, to create a different user experience when travelling in familiar or usual places, or when in a completely unknown environment, where his sense of awareness of things and objects around him must be highly enhanced.

The most common systems use databases populated with points of interest (landmarks) whose description is triggered by the current location of the user and his personal preferences. More advanced systems also try to extract some features from the environment that are not georeferenced. These landmarks may arise because the databases are not frequently updated, or because some unforeseen danger has arisen (hole in the ground, for example), or simply because the user is using the system in a place that does not have a map. In general, these systems based on Computer Vision, work together with location systems. Some newer systems use $3 \mathrm{D}$ cameras to add distance (depth) information to the existing information about the detected elements/landmarks.

Navigation systems for the blind, in general, use technology that is commonly found in traditional navigation systems aimed at people without any special restriction. One example is the routing algorithm known as $\mathrm{A}^{*}$. However, this type of algorithm should be used to make the route calculation, taking into account certain limitations or preferences of the users that are not usually taken into consideration, designing a route that fits the user's preferences, not only considering the shortest path, but possibly also the safest or the most enjoyable. For example, blind people avoid paths with very large open spaces, or certain spaces with protruding structures such as balconies. Due to the noise on crowded sidewalks, they may prefer more quiet gardens, or alternative streets. The blind usually prefer to follow paths accompanied by walls or other guiding elements. They should also be informed in the case of existing dangers in the vicinity of the route. Certain restrictions may even be considered by the routing algorithms to help people with other special needs, such as the existence of stairs for people in wheelchairs.

Regarding the user interface, although there are several ways to interface with the user's natural 
senses, in general the implementation of interface technology should be carefully considered as it may interfere with performing other tasks that the blind users cannot do without. The amount of information to be presented to the user should be carefully filtered as well, as the information capacity of the non-visual senses is much smaller than vision. While navigating to a specific point of interest (macro navigation), the use of natural language must provide timely and accurate directions. For example, in the specific case of blindness, a directional pointing system using the clock position system is more easily understood than using the exact amount of degrees to turn (very accurate, difficult to follow) or relative amounts of left/right ("little to the left" or "hard right", for example, is easy to be understood though results in different interpretations of the quantities). The clock position system is a good example of a natural interface to be used to transmit navigational information, which is sufficiently fast to interpret while maintaining accuracy. The interface must be adaptive in order to differentiate between the different types of use (micro or macro navigation) and combine each state to the level of awareness the user requires in each moment/place, creating a form of contextual awareness. Specific points in which immediate danger may cause harm to the user (e.g., stairs) must bypass all the awareness settings, and the interface must be able to respond as well.

Fusing ideas from these different concepts and techniques, such as the concepts of micro and macro navigation and path planning algorithms, a sophisticated navigation module could combine the inputs from the orientation module (micro navigation) and the route planning techniques (macro navigation) to create a personalized user experience, creating routes that are designed to fit the user's preferences, not only considering the shortest path, but also possibly the safest (according to the type of impairment) or the most enjoyable (for example through a garden instead of a concrete sidewalk).

This review demonstrates that a lot of work already exists in this field, although it is very fragmented. Research in the field of creating assistive technology to assist the orientation and navigation of blind users should follow in the direction of creating integrated products, which use the benefits of different technologies in a combined/integrated way.
In order to do so, the evolution of sensing technology will help achieve massive use.

\section{ACKNOWLEDGMENTS}

The work presented in this paper has been supported by the project CE4blind - Context extraction for the blind using computer vision, with project reference UTAP-EXPL/EEI-SII/0043/2014, by the award "Inclusion and Digital Literacy Prize 2015" promoted by the Portuguese ICT and Society Network, and research grant with reference SFRH/BD/89759/2012. All funding has been granted by the Portuguese Foundation for Science and Technology (FCT).

\section{REFERENCES}

1. WHO (2014) Visual impairment and blindness, Fact Sheet $n^{\circ} 282$. http://www.who.int/mediacentre/factsheets/fs282/en/. Accessed March 16th, 20152015

2. Hakobyan L, Lumsden J, O'Sullivan D, Bartlett H (2013) Mobile assistive technologies for the visually impaired. Survey of Ophthalmology 58 (6):513-528. doi:10.1016/j.survophthal.2012.10.004

3. WHO (2010) International Statistical Classification of Diseases and Related Health Problems (ICD-10). vol 2. WHO,

4. Foley A, Ferri BA (2012) Technology for people, not disabilities: ensuring access and inclusion. Journal of Research in Special Educational Needs 12 (4):192-200. doi:10.1111/j.1471-3802.2011.01230.x

5. Mountain G (2004) Using the Evidence to Develop Quality Assistive Technology Services. Journal of Integrated Care 12 (1):19-26. doi:doi:10.1108/14769018200400005

6. Scherer MJ (2000) Living in the state of stuck: How assistive technology impacts the lives of people with disabilities.

7. Takizawa H, Yamaguchi S, Aoyagi M, Ezaki N, Mizuno S (2013) Kinect Cane : Object Recognition Aids for the Visually Impaired. In: Paja WA, Wilamowski BM (eds) 2013 6th International Conference on Human System Interactions. Conference on Human System Interaction. pp 473478

8. (BRE) BRE (2003) Research Findings No 4: Helping people with sight loss in their homes: housing-related assistive technology. http://www.pocklington-

trust.org.uk/researchandknowledge/publications/op3. Accessed March 10th 2015

9. Montello DR (2009) Cognitive Research in GIScience: Recent Achievements and Future Prospects. Geography Compass 3 (5):1824-1840. doi:10.1111/j.1749-8198.2009.00273.x

10. Jacobson RD (1998) Cognitive mapping without sight: Four preliminary studies of spatial learning. Journal of Environmental Psychology 18 (3):289-305. doi:10.1006/jevp.1998.0098

11. Jacquet C, Bellik Y, Bourda Y (2006) Electronic Locomotion Aids for the Blind: Towards More Assistive Systems. In: Ichalkaranje N, Ichalkaranje A, Jain LC (eds) Intelligent Paradigms for Assistive and Preventive Healthcare, vol 19. Studies in Computational Intelligence. Springer Berlin Heidelberg, pp 133-163. doi:10.1007/11418337_5

12. Passini R, Proulx G, Rainville C (1990) The Spatio-Cognitive Abilities of the Visually Impaired Population. Environment and Behavior 22 (1):91118. doi:10.1177/0013916590221005

13. Song J-W, Yang S-H (2010) Touch your way: haptic sight for visually impaired people to walk with independence.

14. Manduchi R, Kurniawan S, Bagherinia H (2010) Blind guidance using mobile computer vision: a usability study. Paper presented at the 
Proceedings of the 12th international ACM SIGACCESS conference on Computers and accessibility, Orlando, Florida, USA,

15. Fallah N, Apostolopoulos I, Bekris K, Folmer E (2013) Indoor Human Navigation Systems: A Survey. Interacting with Computers 25 (1):21-33. doi:10.1093/iwc/iws010

16. D'Atri E, Medaglia CM, Serbanati A, Ceipidor UB A system to aid blind people in the mobility: A usability test and its results. In: Systems, 2007. ICONS '07. Second International Conference on, 22-28 April 2007 2007. pp 35-35. doi:10.1109/ICONS.2007.7

17. Kulyukin V, Gharpure C, Nicholson J, Osborne G (2006) Robotassisted wayfinding for the visually impaired in structured indoor environments. Auton Robot 21 (1):29-41. doi:10.1007/s10514-006-7223-8 18. Willis S, Helal S RFID information grid for blind navigation and wayfinding. In: Wearable Computers, 2005. Proceedings. Ninth IEEE International Symposium on, 18-21 Oct. 2005 2005. pp 34-37. doi:10.1109/ISWC.2005.46

19. Fernandes H, Filipe V, Costa P, Barroso J (2014) Location based services for the blind supported by RFID technology. Procedia Computer Science 27:2-8

20. Bin D, Haitao Y, Xiaoning Z, Li J The Research on Blind Navigation System Based on RFID. In: Wireless Communications, Networking and Mobile Computing, 2007. WiCom 2007. International Conference on, 2125 Sept. 2007 2007. pp 2058-2061. doi:10.1109/WICOM.2007.514

21. Chumkamon S, Tuvaphanthaphiphat P, Keeratiwintakorn P A blind navigation system using RFID for indoor environments. In: Electrical Engineering/Electronics, Computer, Telecommunications and Information Technology, 2008. ECTI-CON 2008. 5th International Conference on, 1417 May 2008 2008. pp 765-768. doi:10.1109/ECTICON.2008.4600543

22. Fernandes H, Faria J, Paredes H, Barroso J An integrated system for blind day-to-day life autonomy. In: The proceedings of the 13th international ACM SIGACCESS conference on Computers and accessibility, 2011. ACM, pp 225-226

23. Baus J, Krüger A, Wahlster W (2002) A resource-adaptive mobile navigation system. Paper presented at the Proceedings of the 7th international conference on Intelligent user interfaces, San Francisco, California, USA,

24. Tsetsos V, Anagnostopoulos C, Kikiras P, Hadjiefthymiades S (2006) Semantically enriched navigation for indoor environments. Int J Web Grid Serv 2 (4):453-478. doi:10.1504/ijwgs.2006.011714

25. Haosheng H, Gartner G, Schmidt M, Yan L Smart Environment for Ubiquitous Indoor Navigation. In: New Trends in Information and Service Science, 2009. NISS '09. International Conference on, June 30 2009-July 2 2009 2009. pp 176-180. doi:10.1109/NISS.2009.16

26. Chang Y-J, Tsai S-K, Wang T-Y (2008) A context aware handheld wayfinding system for individuals with cognitive impairments. Paper presented at the Proceedings of the 10th international ACM SIGACCESS conference on Computers and accessibility, Halifax, Nova Scotia, Canada, 27. Smailagic A, Martin R Metronaut: a wearable computer with sensing and global communication capabilities. In: Wearable Computers, 1997. Digest of Papers., First International Symposium on, 13-14 Oct. 19971997. pp 116-122. doi:10.1109/ISWC.1997.629927

28. Fischer C, Muthukrishnan K, Hazas M, Gellersen H (2008) Ultrasoundaided pedestrian dead reckoning for indoor navigation. Paper presented at the Proceedings of the first ACM international workshop on Mobile entity localization and tracking in GPS-less environments, San Francisco, California, USA,

29. Höllerer T, Hallaway D, Tinna N, Feiner S Steps toward accommodating variable position tracking accuracy in a mobile augmented reality system. In: Proc. AIMS, 2001. Citeseer, pp 31-37

30. Koide S, Kato M 3-D human navigation system considering various transition preferences. In: Systems, Man and Cybernetics, 2005 IEEE International Conference on, 10-12 Oct. 2005 2005. pp 859-864 Vol. 851. doi:10.1109/ICSMC.2005.1571254

31. Retscher G Pedestrian navigation systems and location-based services. In: 3G Mobile Communication Technologies, 2004. 3G 2004. Fifth IEE International Conference on, 2004 2004. pp 359-363. doi:10.1049/cp:20040696
32. Wu H, Marshall A, Yu W Path planning and following algorithms in an indoor navigation model for visually impaired. In: Internet Monitoring and Protection, 2007. ICIMP 2007. Second International Conference on, 2007. IEEE, pp 38-38

33. Amemiya T, Yamashita J, Hirota K, Hirose M Virtual leading blocks for the deaf-blind: a real-time way-finder by verbal-nonverbal hybrid interface and high-density RFID tag space. In: Virtual Reality, 2004. Proceedings. IEEE, 27-31 March 2004 2004. pp 165-287. doi:10.1109/VR.2004.1310070

34. Brabyn J, Crandall W, Gerrey W Talking signs: a remote signage, solution for the blind, visually impaired and reading disabled. In: Engineering in Medicine and Biology Society, 1993. Proceedings of the 15th Annual International Conference of the IEEE, 1993 1993. pp 13091310. doi:10.1109/IEMBS.1993.979150

35. Priyantha NB, Chakraborty A, Balakrishnan H (2000) The Cricket location-support system. Paper presented at the Proceedings of the 6th annual international conference on Mobile computing and networking, Boston, Massachusetts, USA,

36. Ran L, Helal S, Moore S (2004) Drishti: An Integrated Indoor/Outdoor Blind Navigation System and Service. Paper presented at the Proceedings of the Second IEEE International Conference on Pervasive Computing and Communications (PerCom'04),

37. Zheng P, Ni L (2005) Smart Phone and Next Generation Mobile Computing (Morgan Kaufmann Series in Networking (Paperback)). Morgan Kaufmann Publishers Inc.,

38. Etter R, Specht M (2005) Melodious walkabout - implicit navigation with contextualized personal audio contents. In: In Adj. Proc. Pervasive Computing, 2005. Technology, p 43

39. Helal A, Moore SE, Ramachandran B Drishti: an integrated navigation system for visually impaired and disabled. In: Wearable Computers, 2001. Proceedings. Fifth International Symposium on, 2001 2001. pp 149-156. doi:10.1109/ISWC.2001.962119

40. Huang B, Liu N (2004) Mobile Navigation Guide for the Visually Disabled. Transportation Research Record

41. Arikawa M, Konomi S, Ohnishi K (2007) Navitime: Supporting Pedestrian Navigation in the Real World. Pervasive Computing, IEEE 6 (3):21-29. doi:10.1109/MPRV.2007.61

42. Retscher G, Thienelt M (2004) NAVIO-A navigation and guidance service for pedestrians. Positioning 1 (08)

43. Mok E, Retscher G (2007) Location determination using WiFi fingerprinting versus WiFi trilateration. Journal of Location Based Services 1 (2): $145-159$

44. Faragher R, Harle R (2015) Location fingerprinting with bluetooth low energy beacons. IEEE Journal on Selected Areas in Communications 33 (11):2418-2428

45. Capp M, Picton P (2000) The optophone: an electronic blind aid. Engineering Science \& Education Journal 9 (3):137-143

46. Ancuti C, Ancuti C, Bekaert P (2009) ColEnViSon: Color Enhanced Visual Sonifier A Polyphonic Audio Texture and Salient Scene Analysis. 47. Praveen RG, Paily RP (2013) Blind Navigation Assistance for Visually Impaired Based on Local Depth Hypothesis from a Single Image. Procedia Engineering 64:351-360

48. Tian Y, Yang X, Yi C, Arditi A (2013) Toward a computer visionbased wayfinding aid for blind persons to access unfamiliar indoor environments. Machine vision and applications 24 (3):521-535

49. Yu X, Ganz A Audible vision for the blind and visually impaired in indoor open spaces. In: Engineering in Medicine and Biology Society (EMBC), 2012 Annual International Conference of the IEEE, 2012. IEEE, pp 5110-5113

50. Ruotsalainen L (2013) Vision-Aided Pedestrian Navigation for Challenging GNSS Environments. Suomen geodeettisen laitoksen julkaisuja-Publications of the Finnish Geodetic Institute; 151

51. Aoki H, Schiele B, Pentland A Realtime personal positioning system for a wearable computer. In: Wearable Computers, 1999. Digest of Papers. The Third International Symposium on, 1999. IEEE, pp 37-43

52. Wei Z, Kosecka J Image Based Localization in Urban Environments. In: 3D Data Processing, Visualization, and Transmission, Third 
International Symposium on, 14-16 June 2006 2006. pp 33-40. doi:10.1109/3DPVT.2006.80

53. Hile H, Borriello G (2007) Information Overlay for Camera Phones in Indoor Environments. In: Hightower J, Schiele B, Strang T (eds) Locationand Context-Awareness, vol 4718. Lecture Notes in Computer Science. Springer Berlin Heidelberg, pp 68-84. doi:10.1007/978-3-540-75160-1_5 54. Hide C, Botterill T, Andreotti M Vision-aided IMU for handheld pedestrian navigation. In: Proceedings of the institute of navigation GNSS 2010 Conference, Portland, Oregon, 2010.

55. Chekhlov D, Pupilli M, Mayol W, Calway A Robust Real-Time Visual SLAM Using Scale Prediction and Exemplar Based Feature Description. In: Computer Vision and Pattern Recognition, 2007. CVPR '07. IEEE Conference on, 17-22 June 2007 2007. pp 1-7. doi:10.1109/CVPR.2007.383026

56. Zhang X, Rad AB, Wong Y-K (2012) Sensor Fusion of Monocular Cameras and Laser Rangefinders for Line-Based Simultaneous Localization and Mapping (SLAM) Tasks in Autonomous Mobile Robots. Sensors 12 (1):429-452

57. Mulloni A, Wagner D, Barakonyi I, Schmalstieg D (2009) Indoor Positioning and Navigation with Camera Phones. Pervasive Computing, IEEE 8 (2):22-31. doi:10.1109/MPRV.2009.30

58. Jirawimut R, Prakoonwit S, Cecelja F, Balachandran W (2003) Visual odometer for pedestrian navigation. Instrumentation and Measurement, IEEE Transactions on 52 (4):1166-1173. doi:10.1109/TIM.2003.815996 59. Holzmann C, Hochgatterer M Measuring Distance with Mobile Phones Using Single-Camera Stereo Vision. In: Distributed Computing Systems Workshops (ICDCSW), 2012 32nd International Conference on, 18-21 June 2012 2012. pp 88-93. doi:10.1109/ICDCSW.2012.22

60. Lee S-w, Kang S, Lee S-w (2008) A Walking Guidance System for the Visually Impaired. International Journal of Pattern Recognition and Artificial Intelligence $22 \quad$ (06):1171-1186 doi:doi:10.1142/S0218001408006727

61. Anderson JD, Dah-Jye L, Archibald JK Embedded stereo vision system providing visual guidance to the visually impaired. In: Life Science Systems and Applications Workshop, 2007. LISA 2007. IEEE/NIH, 8-9 Nov. 2007 2007. pp 229-232. doi:10.1109/LSSA.2007.4400926

62. Penedo A, Costa P, Fenandes H, Pereira A, Barroso J Image segmentation in systems of stereo vision for visually impaired people. In: DSAI2009-Proceedings of 2nd International Conference on Software Development for Enhancing Accessibility and Fighting Info-exclusion, 2009. pp 149-156

63. Bourbakis N (2008) Sensing surrounding 3-D space for navigation of the blind. Engineering in Medicine and Biology Magazine, IEEE 27 (1):4955

64. Saez J, Escolano F, Lozano M (2014) Aerial obstacle detection with 3D mobile devices.

65. MicrosoftRobotics (2011) Kinect Sensor. https://msdn.microsoft.com/en-us/library/hh438998.aspx. Accessed April 16th, 2015

66. Henry P, Krainin M, Herbst E, Ren X, Fox D (2014) RGB-D Mapping: Using Depth Cameras for Dense 3D Modeling of Indoor Environments. In: Khatib O, Kumar V, Sukhatme G (eds) Experimental Robotics, vol 79. Springer Tracts in Advanced Robotics. Springer Berlin Heidelberg, pp 477 491. doi:10.1007/978-3-642-28572-1_33

67. Biswas J, Veloso M Depth camera based indoor mobile robot localization and navigation. In: Robotics and Automation (ICRA), 2012 IEEE International Conference on, 2012. IEEE, pp 1697-1702

68. Khoshelham K, Elberink SO (2012) Accuracy and resolution of kinect depth data for indoor mapping applications. Sensors 12 (2):1437-1454 69. Filipe V, Fernandes F, Fernandes H, Sousa A, Paredes H, Barroso J (2012) Blind Navigation Support System based on Microsoft Kinect. $\begin{array}{lllll}\text { Procedia } & \text { Computer } & \text { Science } & 14 & (0): 94-101 .\end{array}$ doi:http://dx.doi.org/10.1016/j.procs.2012.10.011

70. Kammoun S, Parseihian G, Gutierrez O, Brilhault A, Serpa A, Raynal M, Oriola B, Macé M-M, Auvray M, Denis M (2012) Navigation and space perception assistance for the visually impaired: The NAVIG project. Irbm 33 (2):182-189

71. Ross DA, Blasch BB (2002) Development of a wearable computer orientation system. Personal and Ubiquitous Computing 6 (1):49-63

72. Dakopoulos D, Bourbakis NG (2010) Wearable obstacle avoidance electronic travel aids for blind: a survey. Systems, Man, and Cybernetics, Part C: Applications and Reviews, IEEE Transactions on 40 (1):25-35

73. Brilhault A, Kammoun S, Gutierrez O, Truillet P, Jouffrais C Fusion of Artificial Vision and GPS to Improve Blind Pedestrian Positioning. In: New Technologies, Mobility and Security (NTMS), 2011 4th IFIP International Conference on, 7-10 Feb. 2011 2011. pp 1-5. doi:10.1109/NTMS.2011.5721061

74. Moreno M, Shahrabadi S, José J, du Buf JH, Rodrigues JM (2012) Realtime local navigation for the blind: detection of lateral doors and sound interface. Procedia Computer Science 14:74-82

75. Wu J, Zhang J, Yan J, Liu W, Song G (2012) Design of a vibrotactile vest for contour perception. International Journal of Advanced Robotic Systems 9

76. Nagarajan R, Yaacob S, Sainarayanan G Fuzzy-based human vision properties in stereo sonification system for the visually impaired. In: Intelligent Systems and Advanced Manufacturing, 2001. International Society for Optics and Photonics, pp 525-534

77. Murai Y, Kawahara M, Tatsumi H, Araki T, Miyakawa M Congestion recognition for arm navigation. In: Systems Man and Cybernetics (SMC), 2010 IEEE International Conference on, 10-13 Oct. 2010 2010. pp 15301535. doi:10.1109/ICSMC.2010.5642428

78. Coughlan J, Manduchi R (2009) Functional assessment of a camera phone-based wayfinding system operated by blind and visually impaired users. International Journal on Artificial Intelligence Tools 18 (03):379397

79. Ivanchenko V, Coughlan J, Shen H (2008) Crosswatch: a camera phone system for orienting visually impaired pedestrians at traffic intersections. Springer,

80. Ivanchenko V, Coughlan J, Shen H Detecting and locating crosswalks using a camera phone. In: Computer Vision and Pattern Recognition Workshops, 2008. CVPRW'08. IEEE Computer Society Conference on, 2008. IEEE, pp 1-8

81. Karacs K, Radvanyi M, Gorog M, Kusnyerik A, Roska T A mobile visual navigation device: New algorithms for crosswalk and pictogram recognition. In: Applied Sciences in Biomedical and Communication Technologies, 2009. ISABEL 2009. 2nd International Symposium on, 2009. IEEE, pp 1-2

82. Karacs K, Roska T Route number recognition ot Public Transport Vehicles via the Bionic Eyeglass. In: Cellular Neural Networks and Their Applications, 2006. CNNA '06. 10th International Workshop on, 28-30 Aug. 2006 2006. pp 1-6. doi:10.1109/CNNA.2006.341608

83. Guida C, Comanducci D, Colombo C (2011) Automatic bus line number localization and recognition on mobile phones - a computer vision aid for the visually impaired. In: Image Analysis and Processing-ICIAP 2011. Springer, pp 323-332

84. Hasanuzzaman FM, Yang X, Tian Y (2012) Robust and effective component-based banknote recognition for the blind. Systems, Man, and Cybernetics, Part C: Applications and Reviews, IEEE Transactions on 42 (6): 1021-1030

85. Matusiak K, Skulimowski P, Strumillo P Object recognition in a mobile phone application for visually impaired users. In: Human System Interaction (HSI), 2013 The 6th International Conference on, 2013. IEEE, pp 479-484

86. Gomez JD, Mohammed S, Bologna G, Pun T Toward 3D scene understanding via audio-description: Kinect-iPad fusion for the visually impaired. In: The proceedings of the 13th international ACM SIGACCESS conference on Computers and accessibility, 2011. ACM, pp 293-294

87. Takizawa H, Yamaguchi S, Aoyagi M, Ezaki N, Mizuno S Kinect cane: an assistive system for the visually impaired based on three-dimensional 
object recognition. In: System Integration (SII), 2012 IEEE/SICE International Symposium on, 2012. IEEE, pp 740-745

88. Wang S, Pan H, Zhang C, Tian Y (2014) RGB-D image-based detection of stairs, pedestrian crosswalks and traffic signs. Journal of Visual Communication and Image Representation 25 (2):263-272

89. Kang S, Lee S-W Object detection and classification for outdoor walking guidance system. In: Biologically Motivated Computer Vision, 2002. Springer, pp 601-610

90. Kang S, Byun H, Lee S-w (2003) Real-time pedestrian detection using support vector machines. International journal of pattern recognition and artificial intelligence 17 (03):405-416

91. Alghamdi S, van Schyndel R, Khalil I Safe trajectory estimation at a pedestrian crossing to assist visually impaired people. In: Engineering in Medicine and Biology Society (EMBC), 2012 Annual International Conference of the IEEE, 2012. IEEE, pp 5114-5117

92. Muthulakshmi L, Ganesh AB (2012) Bimodal based Environmental Awareness System for visually impaired people. Procedia Engineering 38:1132-1137

93. Downs RM, Stea D (1977) Maps in minds: Reflections on cognitive mapping. HarperCollins Publishers,

94. Foulke E (1982) Perception, cognition and the mobility of blind pedestrians. Spatial abilities: Development and physiological foundations:55-76

95. Loomis JM, Klatzky RL, Golledge RG (2001) Navigating without vision: basic and applied research. Optometry \& Vision Science 78 (5):282-289

96. Kalia AA, Schrater PR, Legge GE (2013) Combining path integration and remembered landmarks when navigating without vision. PloS one 8 (9):e72170

97. Iaria G, Petrides M, Dagher A, Pike B, Bohbot VD (2003) Cognitive strategies dependent on the hippocampus and caudate nucleus in human navigation: variability and change with practice. The journal of neuroscience 23 (13):5945-5952

98. Riehle T, Lichter P, Giudice N An indoor navigation system to support the visually impaired. In: Engineering in Medicine and Biology Society, 2008. EMBS 2008. 30th Annual International Conference of the IEEE, 2008. IEEE, pp 4435-4438

99. Lertlakkhanakul J, Li Y, Choi J, Bu S GongPath: Development of BIM based indoor pedestrian navigation system. In: INC, IMS and IDC, 2009. NCM'09. Fifth International Joint Conference on, 2009. IEEE, pp 382-388 100. Lyardet F, Grimmer J, Muhlhauser M CoINS: context sensitive indoor navigation system. In: Multimedia, 2006. ISM'06. Eighth IEEE International Symposium on, 2006. IEEE, pp 209-218

101. Petrie H, Johnson V, Strothotte T, Raab A, Fritz S, Michel R (1996)

MoBIC: Designing a travel aid for blind and elderly people. Journal of Navigation 49 (01):45-52

102. Dingler T, Lindsay J, Walker BN Learnability of sound cues for environmental features: Auditory icons, earcons, spearcons, and speech. In: Proceedings of the 14th International Conference on Auditory Display, Paris, France, 2008. pp 1-6

103. Frauenberger C, Noistering M (2003) 3D audio interfaces for the blind 104. Walker BN, Lindsay J Navigation performance in a virtual environment with bonephones. In: Proceedings of the International Conference on Auditory Display (ICAD2005), 2005. vol 3. pp 1-26

105. Manduchi R, Coughlan J (2012) (Computer) vision without sight. Communications of the ACM 55 (1):96-104

106. Johnson LA, Higgins CM A navigation aid for the blind using tactilevisual sensory substitution. In: Engineering in Medicine and Biology Society, 2006. EMBS'06. 28th Annual International Conference of the IEEE, 2006. IEEE, pp 6289-6292

107. Pissaloux E, Maingreaud F, Velazquez R, Fontaine E (2006) Concept of the Walking Cognitive Assistance: Experimental Validation. AMSE Int $\mathrm{J}$ on Advances in Modelling (Serie C: Automatic Control) 67:75-86 108. Technology BV (2010) BrainPort Vision Technology Web Page. http://vision.wicab.com. Accessed April 23, 20152010

109. Strumillo $P$ Electronic interfaces aiding the visually impaired in environmental access, mobility and navigation. In: Human System Interactions (HSI), 2010 3rd Conference on, 2010. IEEE, pp 17-24 


\section{Title}

A review of assistive spatial orientation and navigation technologies for the visually impaired

\section{Authors}

Hugo Fernandes*1, Paulo Costa ${ }^{2}$, Vitor Filipe ${ }^{1}$ and João Barroso ${ }^{1}$

${ }^{1}$ School of Science and Technology, INESC TEC and Universidade de Trás-os-

Montes e Alto Douro, Quinta de Prados, Apt.1013, 5001-801, Vila Real, Portugal.

2 Polytechnic Institute of Leiria, Portugal

*Contact author: Hugo Fernandes, School of Science and Technology, INESC TEC and Universidade de Trás-os-Montes e Alto Douro, Quinta de Prados, Apt.1013, 5001-801, Vila Real, Portugal, phone: +351 350 380, email: hugof@utad.pt

\section{Keywords}

blind, review, assistive technology, location, orientation, navigation, computer vision, accessibility. 